\title{
Effects of Quorum Sensing AHL Signaling on the Biological Characteristics of Porcine Derived F4ac+ Enterotoxigenic Escherichia coli
}

\author{
Yang Yang, ${ }^{1,2}$ Ji Shao ${ }^{1,2}$, Mingxu Zhou ${ }^{3,4}$, Qiangde Duan ${ }^{1,2}$, Xinyi Zhang ${ }^{1,2}$ and \\ Guoqiang Zhu' ${ }^{1,2 *}$ \\ ${ }^{1}$ College of Veterinary Medicine, Yangzhou University, Yangzhou 225009, China \\ ${ }^{2}$ Jiangsu Co-Innovation Center for Important Animal Infectious Diseases and Zoonoses, \\ Yangzhou 225009, China \\ ${ }^{3}$ Institute of Veterinary Immunology and Engineering, National Research Center \\ of Engineering and Technology for Veterinary Biologicals, Jiangsu Academy of \\ Agricultural Sciences, Nanjing 210014, China \\ ${ }^{4}$ Jiangsu Key Laboratory for Food Quality and Safety-State Key Laboratory Cultivation \\ Base, Ministry of Science and Technology, Nanjing 210014, China
}

Yang Yang and Ji Shao contributed equally to this work.

\section{A B S T R A C T}

Diarrhea of piglets is an important disease affecting the development of pig industry, while F4 enterotoxigenic Escherichia coli (ETEC) is an important pathogen causing diarrhea of newborn or weaned piglets, which seriously harms animal husbandry and causes huge economic losses. The pathogenicity of E. coli is closely related to its virulence factors, which are strictly regulated in vivo, and the quorum sensing system is involved in this process. To study the effect of quorum sensing signal molecule acyl homoserine lactone (AHL) on the biological characteristics of Enterotoxigenic E. coli (ETEC), gene lasI of Pseudomonas aeruginosa which is responsible for the synthesis of long side-chain AHL (3OC12HSL), and gene yenI of Yersinia enterocolitica which is responsible for the synthesis of short side-chain AHL (C6-HSL), were selected and transformed into ETEC strain C83902, respectively, and endowed with the ability of endogenous synthesis of 3OC12-HSL and C6-HSL. At the same time, $200 \mu \mathrm{mol} / \mathrm{L}$ 3OC12-HSL and C6-HSL were added into the culture medium, respectively, and co-cultured with ETEC strain C83902. ETEC strains were stimulated by exogenous and endogenous long-chain and short-chain AHL molecules, respectively, and their biological characteristics, such as growth characteristics, biofilm formation ability, and adhesion ability to piglets intestinal epithelial cells IPEC-J2 were observed under the influence of the AHLs described above. Real-time PCR was used to detect the transcription levels of genes encoding major subunits of pathogenic factors such as fimbriae, flagella, enterotoxin, adhesin and hemolysin under the influence of the AHLs. The results showed that under the influence of quorum sensing AHLs, the biofilm formation ability and adherence ability of ETEC were significantly reduced, and the expression of $f i C$ and other major virulence factors were regulated. AHL signaling molecules can regulate virulence related characteristics of F4ac+ ETEC.

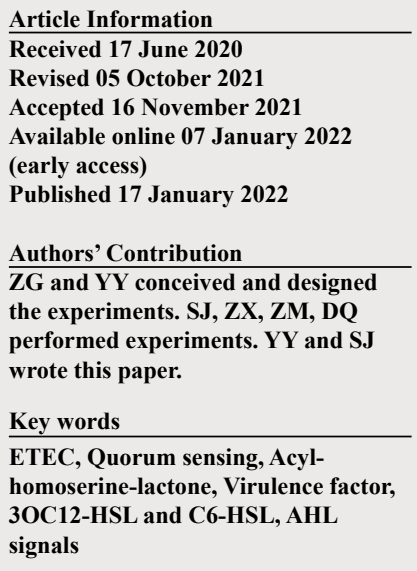

\section{INTRODUCTION}

$\mathrm{P}$ orcine enterotoxigenic Escherichia coli. (ETEC) is an important pathogen that caused diarrhea in newborn and weaned piglets, which causes huge economic losses in pig industry worldwide (Ren et al., 2016). F4ac adhesin positive ETEC bacteria are relatively common in piglet diarrhea and can colonize the intestinal epithelium of piglets via F4ad adhesin, causing piglet diarrhea by releasing

\footnotetext{
Corresponding author: yzgqzhu@yzu.edu.cn 0030-9923/2022/0002-0701\$9.00/0 Copyright 2022 Zoological Society of Pakistan
}

enterotoxins after extensive propagation (Johnson et al., 2009). However, its pathogenic mechanisms have not been fully elucidated.

Quorum sensing (QS) is the way in which specific communication is made between bacteria (Fuqua et al., 1994; Nealson et al., 1970). Bacteria produce signal molecules and release them into the environment. when the bacteria reach a certain population number, and signal molecules in the environment also reach a certain threshold concentration, then the quorum sensing signal can bind to the receptor protein of the bacteria and activate downstream target genes expression to regulate bacterial quorum behaviors, such as bioluminescence, regulation 
of virulence factor secretion, spore formation or biofilm formation, cell differentiation, motility, exopolysaccharide formation, etc. Since the discovery of bacterial quorum sensing in the 1990s, the study of quorum sensing in the regulation of bacterial traits on a population scale has continued to deepen, which is closely related to the synthesis process of a variety of pathogenic bacteria virulence factors. However, the regulatory mechanisms of acyl homoserine lactone (AHL) signaling on enterotoxigenic $E$. coli virulence factors for quorum sensing-1 system has not been fully clarified.

E. coli cannot synthesize AHL molecule on its own, but it has SdiA, the molecular receptor of AHL, which can monitor the AHL signal secreted by adjacent flora in the infection pathway through quorum sensing eavesdropping, so as to further regulate its own virulence (Culler et al., 2018; Styles and Blackwell, 2018; Dyszel et al., 2010; Yakhnin et al., 2011). In previous study of our laboratory, the AHL positive strains in the pig intestine has been screened, and its regulation on the virulence of Shiga toxin-producing E. coli (STEC), Enterohemorrhagic E. coli (EHEC) and avian pathogenic E. coli (APEC) has been verified (Smith et al., 2011; Yang et al., 2013). However, the effect of AHL on the biological characteristics of ETEC needs to be further explored. The previous results revealed that the analysis of the pathogenic mechanism of $E$. coli not only needs to proceed from the regulation mechanism of $E$. coli itself, but also needs to observe the impact of quorum sensing signals released by the adjacent intestinal flora in the path of intestinal infection (Yang et al., 2018b), which can further reveal the changes of the pathogenic mechanism and biological characteristics of ETEC strain in the complex flora environment.

In this study, lasI gene for synthesizing long side chain AHL (3OC12-HSL) in Pseudomonas aeruginosa and yenI gene for synthesizing short side chain AHL (C6HSL) in Yersinia enterocolitica were transformed into ETEC strain C83902 respectively, to construct C83902/ plasI and C83902/pyenI, to construct the recombinant strains with the ability of endogenous synthesis of AHL signal molecule. Meanwhile, $200 \mu \mathrm{mol} / \mathrm{L}$ AHL was added to the culture medium. Effects of AHL from different species and sources upon ETEC were observed.

\section{MATERIALS AND METHODS}

Strains, cell lines and reagents

F4ac+ETEC strain C83902, the AHL signaling bioreporters (Chromobacterium violaceum CV026, Agrobacterium tumefaciens JZA1), and cell line IPEC-J2 of small intestinal epithelial cells from piglets were preserved and provided by our laboratory (Table I). Tryptone and Yeast extract were purchased from Oxoid Inc., DMSO, X-gal, chloramphenicol were purchased from Solarbio. AHLs standards (C6-HSL, 3OC12-HSL) were purchased from Sigma, USA. The Fastpure Plasmid Mini Kit Plasmid Extraction Kit was purchased from Nanjing Vazyme Inc., and 2\% porcine RBCs were purchased from Nanjing Quanyi Biotechnology Co., Ltd.

\section{Construction of recombinant strains}

pyenI, plasI plasmids and vector plasmid pBR322 were respectively electro-transformed into C83902 ETEC strain. Strains were centrifuged after culturing in LB medium for $1 \mathrm{~h}$, and spread on LB plate containing optimal ampicillin. After $24 \mathrm{~h}$ of incubation, single colonies were selected and cultured in LB medium containing ampicillin. After $6 \mathrm{~h}$, plasmids DNA were extracted using the Fast Pure Plasmid Mini Kit and identified by agarose gel electrophoresis.

Table I. Strains and plasmids used in this study.

\begin{tabular}{|c|c|c|}
\hline Strains and plasmids & Details & Reference \\
\hline \multicolumn{3}{|l|}{ Strains } \\
\hline E. coli $\mathrm{C} 83902$ & Wild-type (O8:H19:F4ac+ LT+ STa+ STb+) & Preserved in our laboratory \\
\hline E. coli $\mathrm{C} 83902 /$ pyenI & C83902 carrying pyenI & Constructed in this study \\
\hline E. coli C83902/plasI & C83902 carrying plasI & Constructed in this study \\
\hline E. coli $\mathrm{C} 83902 / \mathrm{pBR}$ & C83902 carrying pBR322 & Constructed in this study \\
\hline A. tumefaciens JZA1 & AHL biosensor & Preserved in our laboratory \\
\hline C. violaceum $\mathrm{CV} 026$ & AHL biosensor & Preserved in our laboratory \\
\hline \multicolumn{3}{|l|}{ Plasmids } \\
\hline pBR322 & Expression vector, $\mathrm{Amp}^{\mathrm{r}}$ & Takara Ltd. \\
\hline pyenI & pBR322 carrying LuxI ORF & Preserved in our laboratory \\
\hline plasI & pBR322 carrying LuxI ORF & Preserved in our laboratory \\
\hline
\end{tabular}




\section{Verification of AHL producing ability}

Plasmid pyenI can synthesize short side chain AHL signal C6-HSL, which can be detected by reporter CV026. Plasmid plasI can synthesize long side chain AHL signal 3OC12-HSL, which can be detected by reporter JZA1.

According to previous method (Chu et al., 2011), strains C83902, C83902/pyenI and CV026 were streaked in parallel on LB plates and cultured at $28^{\circ} \mathrm{C}$ for $24 \mathrm{~h}$. The reporter strain was set as a negative control and C6-HSL as the positive control. The strains C83902, C83902/plasI and JZA1 were cultured in LB medium with $\mathrm{X}$-gal at $28^{\circ} \mathrm{C}$ for $24 \mathrm{~h}$, to observe the color changes; 3OC12-HSL was chosen as the positive control.

\section{The measurement of growth curve}

Single colonies of wild strain C83902, recombinant strains C83902/pBR, C83902/plyenI and C83902/plasI were inoculated into $\mathrm{LB}$ liquid medium respectively, cultured overnight at $37^{\circ} \mathrm{C}$, then transferred to liquid LB broth at the ratio of $1: 100$ the next day. Moreover, the wild type C83902 was transferred to LB liquid medium containing $200 \mu \mathrm{mol} / \mathrm{L}$ C6-HSL and 3OC12-HSL at the ratio of $1: 100.100 \mu \mathrm{L}$ solution samples were collected each hour to detect values of $\mathrm{OD}_{600}$. The average value of three tests were took to draw the growth curve.

\section{Detection of biofilm formation ability}

According to previous method (Coffey and Anderson, 2014), in the endogenous AHL group, C83902, C83902/ pBR, C83902/pyenI, C83902/plasI with OD $_{600}$ of 1.0 were inoculated in biofilm induction medium (tryptone 10.0 $\mathrm{g} / \mathrm{L}$, yeast extract $5.0 \mathrm{~g} / \mathrm{L}, \mathrm{NaCl} 2.5 \mathrm{~g} / \mathrm{L}, \mathrm{KH}_{2} \mathrm{PO}_{4} 3.0 \mathrm{~g} / \mathrm{L}$, $\mathrm{K}_{2} \mathrm{HPO}_{4} 7.0 \mathrm{~g} / \mathrm{L},(\mathrm{NH} 4)_{2} \mathrm{SO}_{4} 2.0 \mathrm{~g} / \mathrm{L}, \mathrm{FeSO}_{4} 0.5 \mathrm{mg} / \mathrm{L}$, $\mathrm{MgSO}_{4} 1.0 \mathrm{~g} / \mathrm{L}$, and thiamine hydrochloride $2.0 \mathrm{~g} / \mathrm{L}$ ). In the exogenous AHL group, the wild type C83902 was transferred to the 3 groups of biofilm induction mediums that without AHL, containing $200 \mu \mathrm{mol} / \mathrm{L}$ C6-HSL, and $200 \mu \mathrm{mol} / \mathrm{L} 3 \mathrm{OC} 12-\mathrm{HSL}$. Medium were then added to 96 well plates, and 12 replicates were employed in each group. Strains were grown at $30{ }^{\circ} \mathrm{C}$ for $48-72 \mathrm{~h}$. The biofilm was detected by crystal violet method and quantified by $\mathrm{OD}_{600}$.

\section{Adherence assays}

According to previous method (Zhou et al., 2013), C83902, C83902/pBR, C83902/pyenI and C83902/plasI with $\mathrm{OD}_{600}$ of 1.0 were added to monolayer IPEC-J2 cells respectively with the multiplicity of infection (MOI) at 100:1, and incubated at $37^{\circ} \mathrm{C}$ for $1 \mathrm{~h}$. Then the cells were washed with PBS for 3 times to remove the non-adhered bacteria, then lysed with $0.5 \%$ tritonX-100 for $20 \mathrm{~min}$. Medium were serial diluted at the ratio of $1: 10$, and finally spread on LB plate. After cultured in $37^{\circ} \mathrm{C}$ overnight,
CFU from each group were counted.

The wild strain C83902 was transferred to LB medium of $200 \mu \mathrm{mol} / \mathrm{L}$ C6-HSL or 3OC12-HSL. Strains were cultured at $37{ }^{\circ} \mathrm{C}$ for $6 \mathrm{~h}$, and then were adjusted to $\mathrm{OD} 600=1.0$. Each group was centrifuged at $5000 \mathrm{r} / \mathrm{min}$ for $10 \mathrm{~min}$, the supernatant was discarded, and bacterial precipitation was resuspended in DMEM at with 200 $\mu \mathrm{mol} / \mathrm{L}$ C6-HSL or 3OC12-HSL. Then adherence assay was carry out.

\section{Motility test}

In the endogenous AHL group, C83902, C83902/ pBR, C83902/pyenI, C83902/plasI with $\mathrm{OD}_{600}$ at 1.0 were inoculated in LB medium at the ratio of $1: 100$, cultured at $37^{\circ} \mathrm{C}$ until the logarithmic phase., then diluted to $\mathrm{OD}_{600}$ at 1.0. $1 \mu \mathrm{L}$ solution of each strain was added in the middle of $0.3 \%$ semi-solid plates. After incubation at $37^{\circ} \mathrm{C}$ for about $18 \mathrm{~h}$, the diameter of bacterial motion halos was measured.

In the exogenous AHL group, wild type C83902 was transferred into LB medium, cultured overnight, took $1 \mu \mathrm{L}$ bacterial solution and added to $0.3 \%$ semi-solid plate (one group without AHL, one group containing $200 \mu \mathrm{mol} / \mathrm{L}$ C6HSL, one group with 3OC12-HSL), After incubation at 37 ${ }^{\circ} \mathrm{C}$ for about $18 \mathrm{~h}$, the diameter of bacterial motion halos were measured.

\section{Hemolytic activity test}

The $2 \%$ porcine erythrocytes were purchased from Nanjing Quanyi Biotechnology Co., Ltd. The strains were cultured to logarithmic phase, then $1 \mathrm{~mL}$ bacterial solution was collected, centrifuged at $10000 \mathrm{rpm}$ for $10 \mathrm{~min}$, and the supernatant was filtered use $0.22 \mu \mathrm{m}$ filtration membrane. $100 \mu \mathrm{L}$ filtered supernatant was collected in each group and mixed with $100 \mu \mathrm{L}$ porcine erythrocytes. Solutions were incubated at $37{ }^{\circ} \mathrm{C}$ for $2 \mathrm{~h}$ and at $4{ }^{\circ} \mathrm{C}$ overnight. After the precipitation of insoluble erythrocyte, $100 \mu \mathrm{L}$ supernatant from each group was took for $\mathrm{OD}_{400}$ value measurement.

\section{Detection the expression level of virulence genes}

According to previous method (Croxen and Finlay, 2010), the bacterial virulence genes (type I fimbria subunit fim $H$ and $f i m A$, flagellum subunit $f i C$, hemolysin hlyA, sfm fimbria subunit $s f m H$, and F4 fimbria subunit fae $G$ ) were selected and real-time PCR (rtPCR) was performed. Primers are shown in Table II.

The above strains were cultured to the logarithmic prophase $\left(\mathrm{OD}_{600}=1.00\right), 1 \mathrm{~mL}$ of bacterial solution was taken to extract mRNA, and was transcripted into cDNA by TaKaRa Reverse Transcription Kit, and RT-PCR was carried out with corresponding cDNA as template. RT-PCR reaction system $(20 \mu \mathrm{L})$ : SYBR Green PCR Master Mix 10 
$\mu \mathrm{L}, 1 \mu \mathrm{L}$ of each upstream and downstream primers $(0.5$ $\mu \mathrm{mol} / \mathrm{L}), 3 \mu \mathrm{L}$ cDNA template $(1.5 \mathrm{ng} / \mu \mathrm{L})$, complement with $\mathrm{ddH}_{2} \mathrm{O}$ up to $20 \mu \mathrm{L}$. PCR reaction conditions: $95^{\circ} \mathrm{C}$ for $2 \mathrm{~min} ; 95^{\circ} \mathrm{C}$ for $5 \mathrm{~s}, 60^{\circ} \mathrm{C}$ for $10 \mathrm{~s}, 40$ cycles. The expression level of the target genes was analyzed and calculated by $2^{-\triangle \triangle \mathrm{CT}}$ method.

Table II. Primers used in this study.

\begin{aligned} & \hline Primers Sequencing (5'-3') \\ & \hline faeG-RT-F ACTCAGAAAACCTGATGGTGAAACT \\ & R CCCCACCTCTCCCTAACACA \\ & sfmH-RT-F TGTGACATCAACGCCTTACGA \\ & $\mathrm{R}$ ATGCGCCGATATCACCAAAA \\ &$i p f A-\mathrm{RT}-\mathrm{F}$ AACCGGCGTTGGTATTCGTA \\ & $\mathrm{R}$ TCATTTCAGCCTTGCCTTCA \\ & fimH-RT-F GGCTGCGATGTTTCTGCTC \\ & $\mathrm{R}$ CCCCAGGTTTTGGCTTTTC \\ &$h l y A-\mathrm{RT}-\mathrm{F}$ TTGCACTCAGCAGGACAAAG \\ & $\mathrm{R}$ GGTGAGGCCAATGAGTTTCT \\ & gap $A$-RT-F CGTTAAAGGCGCTAACTTCG \\ & $\mathrm{R}$ ACGGTGGTCATCAGACCTTC \\ & fic-RT-F TCGACAAATTCCGCTCCTC \\ & $\mathrm{R}$ GGTTGGTGGTGGTGTTGTTC \\ & \hline\end{aligned}

\section{RESULTS}

Verification of recombinant strains and the growth curves The plasmids of C83902/pBR, C83902/plyenI and C83902/plasI were extracted from the recombinant strain, and verified by agarose gel electrophoresis. The results of AHL signal molecule detection showed that, the contact region between recombinant strain C83902/ plasI and CV026 turned purple, which was consistent with the positive control (Fig. 1A). The recombinant strain C83902/plasI induce blue color in reporter strain JAZ1, which was consistent with the positive control, while the color with wild type C83902 did not change (Fig. 1B). It was proved that the recombinant strains with the ability to endogenously synthesize C6-HSL and 3OC12-HSL were successfully constructed. The control C83902/pBR that transformed with empty vector plasmid pBR322 could not induce the color change in CV026 or JZA1. It can be seen from the growth curves that both endogenous synthesis and exogenous addition of AHL had no significant effect on the growth of C83902 in aerobic environment (Fig. 2).

\section{Results of biofilm formation}

The results showed that under endogenous AHL, the biofilm forming ability of recombinant strains C83902/ pyenI and C83902/plasI decreased by $24.5 \%$ and $33.5 \%$ respectively, compared with the wild type C83902 (Fig. 3A). The biofilm forming ability of strain C83902 decreased by $47.6 \%$ and $25 \% \%$ when exogenous C6-HSL and 3OC12-HSL was added, respectively (Fig. 3B).
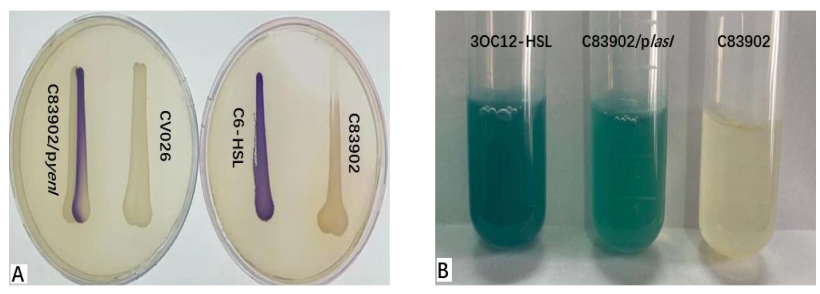

Fig. 1. The signal molecule detection of AHL. A, Detection the ability of C83902/pyenI to synthesize short side chain AHL by reporter strain CV026. B, Detection the ability of C83902/plasI to synthesize long side chain AHL by reporter strain JZA1. C6-HSL and 3OC12-HSL were used as positive controls.

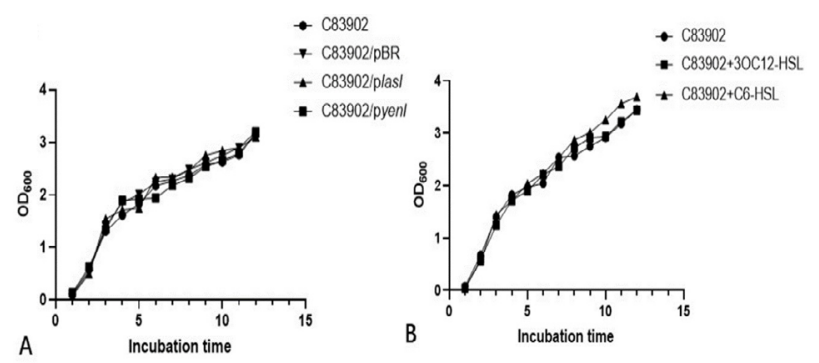

Fig. 2. The growth curves of $\mathrm{C} 83902$ and its recombinant strains under endogenous (A), and exogenous AHLs influence (B).
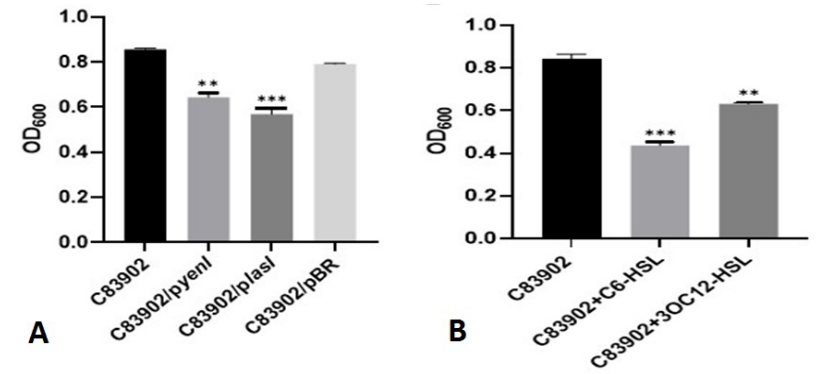

Fig. 3. Quantitative test of biofilm formation of C 83902 and its recombinant strains under endogenous (A) and exogenous AHLs influence (B).

\section{Adherence assays}

The results showed that, under endogenous AHL, the adhesion ability of recombinant strain C83902/pyenI and C83902/plasI decreased by 20.9 and $42.9 \%$ respectively 
compared with wild type C83902 (Fig. 4A). When exogenous C6-HSL and 3OC12-HSL were added, the adhesion ability of C83902 to IPEC-J2 cells decreased by 36.6 and $19.5 \%$, respectively (Fig. 4B).
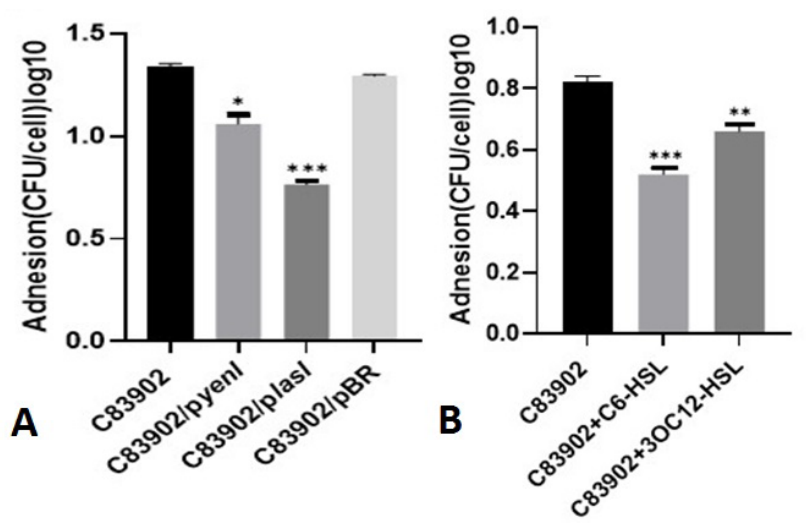

Fig. 4. The adherence ability of IPEC-J2 cells of C 83902 and its recombinant strain under endogenous (A), and exogenous addition of AHL (B). **, $P<0.01$; ***, $P<0.001$.

\section{The motility of strains}

The results of motility test showed that under the effect of endogenous AHL, the diameter of motility circle of recombinant strains C83902/pyenI and C83902/ plasI decreased by $15.5 \%$ and $21.4 \%$, respectively. When exogenous C6-HSL and 3OC12-HSL was added, the motility circle diameter of strain C83902 decreased by $12.3 \%$ and $16.1 \%$, respectively.

\section{Hemolytic activity}

The results showed that under the endogenous AHL, the hemolytic activity of recombinant strains C83902/ pyenI and C83902/plasI increased by $12.5 \%$ and $11.4 \%$, respectively (Fig. 5A); while the hemolytic activity of strain C83902 increased by $13.2 \%$ and $10.2 \%$, respectively when exogenous C6-HSL and 3OC12-HSL was added.

\section{Detection of virulence gene expression}

The results showed that, compared with wild type C83902, the expression of $h l y A$, sfmH, fimH and ipfA in recombinant strain C83902/pyenI increased by 25, 27, 10 and $12 \%$, respectively, while the expression of $f i C$, fim $\mathrm{A}$ and $f a e G$ decreased by 54,21 and $24 \%$. The expression of hlyA, sfmH, fimH, ipfA and fae $G$ increased by 69,79 , 9,25 and $9 \%$, respectively after addition of $200 \mu \mathrm{mol} / \mathrm{L}$ exogenous C6-HSL, while the expression of $\mathrm{fliC}$ and $\mathrm{fimA}$ decreased by 16 and 15\% (Fig. 5A).

Compared with wild type $\mathrm{C} 83902$, the expression of hlyA, sfmH, stfG and ipfA in recombinant strain C83902/
plasI increased by $85,14,7$ and $8 \%$, respectively, while the expression of $f i m H, f i C, f i m A$ and $f a e G$ decreased by 19, 44, 29 and14\%. And when addition of $200 \mu \mathrm{mol} / \mathrm{L}$ exogenous 3OC12-HSL, the expressions of hly A, sfmH, stf $G$ and fim $A$ increased by $84,23,72$ and $31 \%$, while the expressions of fimH, fliC, stfG and ipfA decreased by 19 , 27, 84 and 74\%, respectively (Fig. 5B).
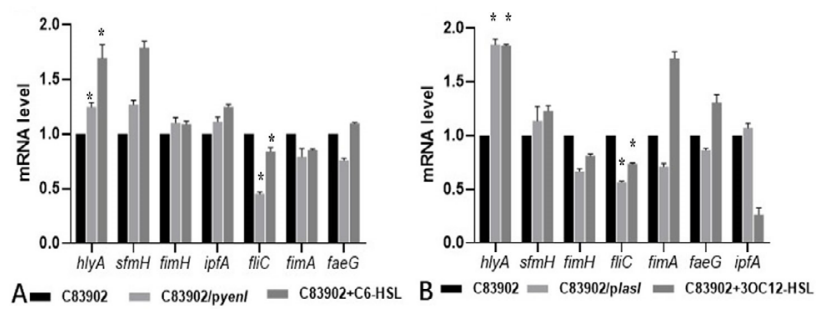

Fig. 5. Transcriptional levels of virulence related genes of $\mathrm{C} 83902$ and its recombinant strains endogenous and exogenous C6-HSL (A) and OC12-HSL (B). * $P<0.05$

\section{DISCUSSION}

F4ac ETEC is a widely prevalent pathogen causing diarrhea in piglets, which causes huge economic losses in pig industry all over the world (Sugiharto et al., 2012; Van den Broeck et al., 2002). The pathogenicity and virulence regulation mechanism of ETEC have been well deeply studied (Luppi, 2017). However, these studies usually focus on ETEC, observing that ETEC regulates virulence factors through its own pathway and mediates the pathogenic effect on the host, while ignoring the impact on ETEC from the perspective of adjacent and intestinal flora (Smith et al., 2008). Since the discovery of bacterial quorum sensing in the 1990 s, the study of quorum sensing in population scale regulation of bacterial traits has continued to be deepen, and it is closely related to the expression process of a variety of pathogenic bacteria virulence factors. It is usually considered that $E$. coli only has quorum sensing-II system, but does not have a complete quorum sensing-1 system, so it cannot synthesize AHLs, the signal molecular of quorum sensing-1. E. coli encodes AHL receptor protein SdiA, which can sense and respond to AHL signals produced by other bacteria, so as to regulate $E$. coli biological functions (Smith et al., 2011; Yakhnin et al., 2011). The research of our laboratory has confirmed the virulence regulation function of AHL in Shiga toxin producing Escherichia coli STEC, EHEC (enterohemorrhagic E. coli) and APEC (avian pathogenic Escherichia coli) (Yang et al., 2013, 2014, 2018a, b). Mechanism of AHLs upon ETEC is still unknown, through which ETEC sense and respond to AHL synthesized by adjacent flora in intestinal environment, so as to start quorum sensing eavesdropping to change its 
own characteristics.

In this study, lasI and yenI, the homologous genes of quorum sensing luxI, were transformed into F4ac + ETEC $\mathrm{C} 83902$ to confer it with the ability of endogenous synthesis of C6-HSL and 3OC12-HSL. These two AHL signals are more important in more than 30 kinds of AHL in nature, and they have been studied in depth. These two types of AHL can represent short-chain side-chain AHL and longchain side-chain AHL molecules respectively through the difference in the number of side chain carbon atoms. Using these two different AHL molecules with different traits can reveal the AHL function more comprehensively. In this study, CV026 which can detect short side chain of AHL, and JZA1 which can detect long side chain of AHL, were used to prove the successful construction of the recombinant strains. Moreover, by adding C6-HSL and 3OC12-HSL to the culture medium, 4 different culture conditions (endogenous, exogenous of short side chain or long side chain of AHL) were formed, so as to more comprehensively demonstrate the virulence regulation effect of AHL on F4ac ETEC strain.

The results showed that, different sources and types of AHL had no significant effect on the growth curve of F4ac+ETEC. Biofilm (BF) is a microbial population formed by the adhesion of bacterial surface polysaccharides, proteins and nucleic acids to abiotic or bioactive surfaces (Rabin et al., 2015). It can improve the tolerance of bacteria to antibiotics, environmental pressure and host immune attack (Płusa, 2019; Sharma et al., 2019). It is an important factor that cannot be ignored in the process of ETEC infection. The biofilm forming ability of ETEC strain decreased significantly under the effect of different sources and different kinds of AHL. In most strains of Pseudomonas aeruginosa, the expression of QS factor stimulates biofilm formation, while in $E$. coli, it shows a unique biofilm inhibition phenomenon under AHL (Li et al., 2015; Yang et al., 2013). There are many factors affecting biofilm, researchers generally believe that biofilm synthesis in E. coli is related to the expression of flagella. During biofilm formation, flagella can help bacteria adhere to the surface of solid medium and help bacteria gather and form biofilm through movement chemotaxis.

Flagella is a very important virulence factor of $E$. coli. It not only provides motility, but also participates in the adhesion, colonization and invasion of bacteria to host cells (Zhou et al., 2015). In this study, the changes of adhesion ability of ETEC to porcine small intestinal epithelial cells IPEC-J2 under AHL were detected by adherence assay. The adhesion ability of ETEC strains decreased significantly under different sources or types of AHL, and the decrease of adhesion ability may come from the inhibition of the expression level of adhesion factors such as flagella by AHL. The results of flagella motility test showed that ETEC motility was inhibited, which was consistent with the results of adherence test.

Flagella usually interacts with the expression of other virulence factors. Virulence factors of ETEC mainly include fimbria, flagella, enterotoxin, adhesin, hemolysin, etc. (Nagy et al., 1996). In this study, the expression level of virulence factor related genes such as flagella was tested by $\mathrm{qPCR}$. After endogenous synthesis and exogenous addition of C6-HSL, the gene expression levels of $h l y A$, $s f m H$, fimH, stfG and ipfA increased, while the expression levels of $\mathrm{AiC}$ and fimA decreased. Endogenous C6-HSL inhibited faeG expression, while exogenous C6-HSL activated fae $G$ expression. After endogenous synthesis and exogenous addition of 3OC12-HSL, the expression of $h l y A$ and $s f m H$ increased, while the expression of $f i m H$ and $f i C$ decreased. Endogenous of 3OC12-HSL up-regulated the expression of $s t f G$ and $i p f A$, but inhibited the expression of fim $A$ and faeG. Its regulatory effect was opposite to that of exogenous 3OC12-HSL. Among these virulence factors, under different sources or different kinds of AHL, the expression level of flagella gene $\mathrm{fliC}$ and hemolysin gene hlyA maintained the same trend and the difference is significant, suggesting that AHL may inhibit flagella expression and activate the synthesis of $\alpha$-hemolysin. The inhibition effect on the synthesis of flagella by AHL may be closely related to the changes of virulence related biological characters such as biofilm formation and decreased adhesion of F4ac ETEC. $\alpha$-hemolysin is a Repeats in toxin (RTX) protein. It has cytolytic activity and cytotoxicity to most mammalian cells such as erythrocytes, granulocytes, monocytes and endothelial cells (Askari et al., 2016). It can integrate into the host cell membrane, affect the stability of cell membrane and induce lysis, thus it is an important virulence factor for ETEC extraintestinal infection and penetrating the intestinal vascular barrier (Burgos and Beutin, 2010). Hemolytic activity test showed that $\alpha$-hemolysin activity was up-regulated by AHL. This study found that AHL is related to hemolysin for the first time, and its regulation mechanism urgently needs further analysis.

\section{CONCLUSION}

In conclusion, for quorum sensing-1 system, this study selected two different kinds of important AHL signals, verified the regulation of AHL on virulence related biological characteristics of F4ac ETEC by endogenous synthesis and exogenous addition of $\mathrm{AHL}$, and revealed AHL effects on flagella expression, biofilm formation, adhesion ability and hemolysin synthesis. It is suggested that the adjacent flora in the ETEC infection pathway 
can interfere with ETEC biological characteristics from the population scale, which lays a foundation for the comprehensive disclosure of the pathogenic pathway of ETEC.

\section{ACKNOWLEDGEMENT}

This study was supported by grants from the Chinese National Science Foundation Grants (Nos. 31972708, 31502075, 31873010, 31672579), "13th Five-Year" National Key Development Program (2016YFD0501000), the Priority Academic Program Development of Jiangsu Higher Education Institutions.

\section{Ethical compliance}

There are no researches conducted on animals or humans.

\section{Statement of conflict of interest}

The authors have declared no conflict of interest.

\section{REFERENCES}

Askari, B.M., Morabito, S., Najafifar, A. and Mazandarani, E., 2016. Molecular characterization of enterohemorrhagic Escherichia coli hemolysin gene (Ehec-Hlya)-harboring isolates from cattle reveals a diverse origin and hybrid diarrheagenic strains. Infect. Genet. Evol., 39: 342-348. https:// doi.org/10.1016/j.meegid.2016.02.002

Burgos, Y. and Beutin, L., 2010. Common origin of plasmid encoded alpha-hemolysin genes in Escherichia coli. BMC Microbiol., 10: 193. https:// doi.org/10.1186/1471-2180-10-193

Chu, W., Vattem, D.A., Maitin, V., Barnes, M.B. and Mclean, R.J.C., 2011. Bioassays of quorum sensing compounds using agrobacterium tumefaciens and chromobacterium violaceum. Methods Mol. Biol., 692: 3-19. https://doi.org/10.1007/978-1-60761971-0 1

Coffey, B.M. and Anderson, G.G., 2014. Biofilm formation in the 96-well microtiter plate. Pseudomonas: Methods Protocols, 1149: 631-641. https://doi.org/10.1007/978-1-4939-0473-0_48

Croxen, M.A. and Finlay, B.B., 2010. Molecular mechanisms of Escherichia coli pathogenicity. Nat. Rev. Microbiol., 8: 26-38. https://doi.org/10.1038/ nrmicro2265

Culler, H.F., Couto, S.C.F., Higa, J.S., Ruiz, R.M., Yang, M.J., Bueris, V., Franzolin, M.R. and Sircili, M.P., 2018. Role of sdia on biofilm formation by atypical enteropathogenic Escherichia coli. Genes,
9: 253-255. https://doi.org/10.3390/genes9050253

Dyszel, J.L., Soares, J.A., Swearingen, M.C., Lindsay, A., Smith, J.N. and Ahmer, B.M.M., 2010. E. coli $\mathrm{K}-12$ and Ehec genes regulated by Sdia. PLoS One, 5: e8946. https://doi.org/10.1371/journal. pone. 0008946

Fuqua, W.C., Winans, S.C. and Greenberg, E.P., 1994. Quorum sensing in bacteria: The luxr-luxi family of cell density-responsive transcriptional regulators. J. Bact., 176: 269-275. https://doi.org/10.1128/ jb.176.2.269-275.1994

Johnson, A.M., Kaushik, R.S., Rotella, N.J. and Hardwidge, P.R., 2009. Enterotoxigenic Escherichia coli modulates host intestinal cell membrane asymmetry and metabolic activity. Infect. Immunol., 77: 341-347. https://doi. org/10.1128/IAI.01097-08

Li, H., Li, X., Wang, Z., Fu, Y., Ai, Q., Dong, Y. and Yu, J., 2015. Autoinducer-2 regulates pseudomonas aeruginosa pao1 biofilm formation and virulence production in a dose-dependent manner. $B M C$ Microbiol., 15: 192. https://doi.org/10.1186/ s12866-015-0529-y

Luppi, A., 2017. Swine enteric colibacillosis: Diagnosis, therapy and antimicrobial resistance. Porc. Hlth. Manage., 3: 16. https://doi.org/10.1186/s40813017-0063-4

Nagy, B., Nagy, G., Meder, M. and Mocsári, E., 1996. Enterotoxigenic Escherichia coli, rotavirus, porcine epidemic diarrhoea virus, adenovirus and calici-like virus in porcine postweaning diarrhoea in Hungary. Acta Vet. Hung., 44: 9-19.

Nealson, K.H., Platt, T. and Hastings, J.W., 1970. Cellular control of the synthesis and activity of the bacterial luminescent system. J. Bact., 104: 313-322. https://doi.org/10.1128/jb.104.1.313322.1970

Płusa, T., 2019. The importance of biofilm in the context of increasing bacterial resistance to antibiotics. Pol. Merkuriusz Lek., 47: 197-202.

Rabin, N., Zheng, Y., Opoku-Temeng, C., Du, Y., Bonsu, E. and Sintim, H.O., 2015. Biofilm formation mechanisms and targets for developing antibiofilm agents. Future Med. Chem., 7: 493-512. https://doi. org/10.4155/fmc.15.6

Ren, W., Yin, J., Chen, S., Duan, J., Liu, G., Li, T., Li, N., Peng, Y., Tan, B. and Yin, Y., 2016. Proteome analysis for the global proteins in the jejunum tissues of enterotoxigenic Escherichia coli infected piglets. Sci. Rep., 6: 1-9. https://doi.org/10.1038/ srep25640

Sharma, D., Misba, L. and Khan, A.U., 2019. Antibiotics 
versus biofilm: An emerging battleground in microbial communities. Antimicrob. Resist. Infect. Contr., 8: 76. https://doi.org/10.1186/s13756-0190533-3

Smith, J.L., Fratamico, P.M. and Yan, X., 2011. Eavesdropping by bacteria: The role of Sdia in Escherichia coli and salmonella enterica serovar typhimurium quorum sensing. Foodborne Pathog. Dis., 8: 169-178. https://doi.org/10.1089/ fpd.2010.0651

Smith, J.N., Dyszel, J.L., Soares, J.A., Ellermeier, C.D., Altier, C., Lawhon, S.D., Adams, L.G., Konjufca, V., Curtiss, R., Slauch, J.M. and Ahmer, B.M., 2008. Sdia, a N-acylhomoserine lactone receptor, becomes active during the transit of salmonella enterica through the gastrointestinal tract of turtles. PLoS One, 3: e2826. https://doi.org/10.1371/ journal.pone.0002826

Styles, M.J. and Blackwell, H.E., 2018. Non-native autoinducer analogs capable of modulating the Sdia quorum sensing receptor in salmonella enterica serovar typhimurium. Beilstein J. Org. Chem., 14: 2651-2664. https://doi.org/10.3762/bjoc.14.243

Sugiharto, S., Hedemann, M.S., Jensen, B.B. and Lauridsen, C., 2012. Diarrhea-like condition and intestinal mucosal responses in susceptible homozygous and heterozygous $\mathrm{F} 4 \mathrm{r}+$ pigs challenged with enterotoxigenic Escherichia coli. J. Anim. Sci., 90 (Suppl 4): 281-283. https://doi. org/10.2527/jas.53840

Van den Broeck, W., Bouchaut, H., Cox, E. and Goddeeris, B.M., 2002. F4 receptor-independent priming of the systemic immune system of pigs by low oral doses of F4 fimbriae. Vet. Immunol. Immunopathol., 85: 171-178. https://doi. org/10.1016/S0165-2427(01)00429-9

Yakhnin, H., Baker, C.S., Berezin, I., Evangelista, M.A., Rassin, A., Romeo, T. and Babitzke, P., 2011.
Csra represses translation of Sdia, which encodes the N-Acylhomoserine-L-Lactone receptor of Escherichia coli, by binding exclusively within the coding region of Sdia Mrna. J. Bact., 193: 61626170. https://doi.org/10.1128/JB.05975-11

Yang, Y., Liu, Y., Zhou, M. and Zhu, G., 2018a. Both quorum sensing (Qs)-I and Ii systems regulate Escherichia coli flagellin expression. Pakistan J. Zool., 50: 1807-1813. https://doi.org/10.17582/ journal.pjz/2018.50.5.1807.1813

Yang, Y., Yao, F., Zhou, M., Zhu, J., Zhang, X., Bao, W., Wu, S., Hardwidge, P.R. and Zhu, G., 2013. F18ab Escherichia coli flagella expression is regulated by acyl-homoserine lactone and contributes to bacterial virulence. Front. Cell. Infect. Microbiol., 165: 378383. https://doi.org/10.1016/j.vetmic.2013.04.020

Yang, Y., Zhou, M., Hardwidge, P.R., Cui, H. and Zhu, G., 2018b. Isolation and characterization of $\mathrm{N}$-Acyl homoserine lactone-producing bacteria from cattle rumen and swine intestines. Front. Cell Infect. Microbiol., 8: 155. https://doi.org/10.3389/ fcimb.2018.00155

Yang, Y., Zhou, M., Hou, H., Zhu, J., Yao, F., Zhang, X., Zhu, X., Hardwidge, P.R. and Zhu, G., 2014. Quorum-sensing gene luxs regulates flagella expression and shiga-like toxin production in F18ab Escherichia coli. Can. J. Microbiol., 60: 355-361. https://doi.org/10.1139/cjm-2014-0178

Zhou, M., Duan, Q., Zhu, X., Guo, Z. and Research, Y.L.J.V., 2013. Both flagella and F4 fimbriae from F4ac+ enterotoxigenic Escherichia coli contribute to attachment to Ipec-J2 cells in vitro. Vet. Res., 44: 1-6. https://doi.org/10.1186/1297-9716-44-30

Zhou, M., Yang, Y., Chen, P., Hu, H., Hardwidge, P.R. and Zhu, G., 2015. More than a locomotive organelle: flagella in Escherichia coli. Appl. Microbiol. Biotechnol., 99: 8883-8890. https://doi. org/10.1007/s00253-015-6946-X 